Gen Nishimura - Tomonobu Hasegawa - Kyoko Sugii

Kaiichiro Tsuyama • Nobutake Matsuo

\title{
Joint laxity, vitreoretinal degeneration, facial abnormalities, and generalized skeletal alterations: A new syndrome?
}

Received: February 5, 1998 / Accepted: March 7, 1998

\begin{abstract}
A Japanese girl with a hitherto unknown combination of malformations is reported. The cardinal features included hyperextensibility of the joints, vitreoretinal degeneration with cataracts, and facial abnormalities, comprising hypertelorism, prominent eyes, downslanting of the palpebral fissures, mid-face recession with a short nose, deformed auricles, and microretrognathia with a high arched palate. Skeletal survey revealed multiple wormian bones, hypoplastic facial bones and mandible, narrow thorax with wavy ribs, narrow ilia, and coxa valga with slight broadening of the proximal femora, findings of which were individually minor, but the assemblage of which assisted in the syndromic identification. Although skin biopsy did not contribute to the causal clarification, it was tempting to speculate that the syndromic constellation of the present disorder resulted from an underlying defect of connective tissues.
\end{abstract}

Key words Vitreoretinal degeneration - Cataracts · Joint laxity $\cdot$ Mid-face hypoplasia $\cdot$ Skeletal abnormalities $\cdot$ Connective tissue disorder

\section{Introduction}

Joint laxity, vitreoretinal degeneration, and generalized skeletal abnormalities individually represent major syndromic constituents of heritable connective tissue disorders, as in Ehlers-Danlos syndrome, type II collagenopathies, and a

G. Nishimura $(\bowtie)$

Department of Radiology, Dokkyo University School of Medicine, 880 Kitakobayashi, Mibu, Shimotsuga-gun, Tochigi 321-02, Japan

Tel. +81-282-87-2171; Fax +81-282-86-4940

e-mail: gen-n@dokkyomed.ac.jp

T. Hasegawa $\cdot$ K. Sugii · N. Matsuo

Department of Pediatrics, Keio University School of Medicine,

Tokyo, Japan

K. Tsuyama

Department of Ophthalmology, Hiratsuka City Hospital, Kanagawa, Japan variety of bone dysplasias, respectively. We recently encountered a Japanese girl with a combination of these abnormalities concurrent with facial dysmorphism. The clinical features of this patient are described in detail to propose a previously unrecognized connective tissue disorder, and the differential diagnosis of this case is discussed.

\section{Case report}

The patient was a Japanese girl who was the second child of a healthy, unrelated, Japanese couple, a 28-year-old father and a 26-year-old mother. The mother had experienced a miscarriage. The elder sister of the patient was healthy. The family history was unremarkable, other than the fact that the parents and elder sister of the patient had undergone a conservative measure for developmental dysplasia of the hip. The patient was vaginally delivered at 40 weeks' gestation after an unremarkable pregnancy. Birth weight was $2900 \mathrm{~g}(-0.5 \mathrm{SD})$. She underwent an orthotic measure for unilateral developmental dysplasia of the hip. She was noted to have generalized hyperextensibility of the joints during infancy. Psychomotor development was normal, as was physical growth. Microscopic hematuria was noted at a general health check at age 3 years. The patient was referred for the evaluation of microscopic hematuria at age 5 $3 / 12$ years. Height was $112.8 \mathrm{~cm}(+1.3 \mathrm{SD})$, and weight $17.3 \mathrm{~kg}(-0.3 \mathrm{SD})$. She exhibited craniofacial dysmorphism, including hypertelorism, downslanting of the palpebral fissures, mild exophthalmos, mid-face recession with a short nose, deformed auricles, and microretrognathia with a high arched palate (Fig. 1a,b). There was striking hyperextensibility of the large and small joints, which gave rise to genu recarvatum and severe flatfeet (Fig. 2a,b). No skin elasticity was found. Ophthalmologic examination revealed hymyopia: the axial length of the right eye was $26.99 \mathrm{~mm}$, and that of the left $28.13 \mathrm{~mm}$; right visual acuity $(\mathrm{RV})=0.09\left(0.7 \times-9.75^{\mathrm{D}}\right)$, left visual acuity $(\mathrm{LV})=0.08$ $\left(0.5 \times-16.0^{\mathrm{D}}\right)$. Bilateral cortical cataracts were evident. Vitreoretinal abnormalities, comprising cloud in the periph- 
a

b
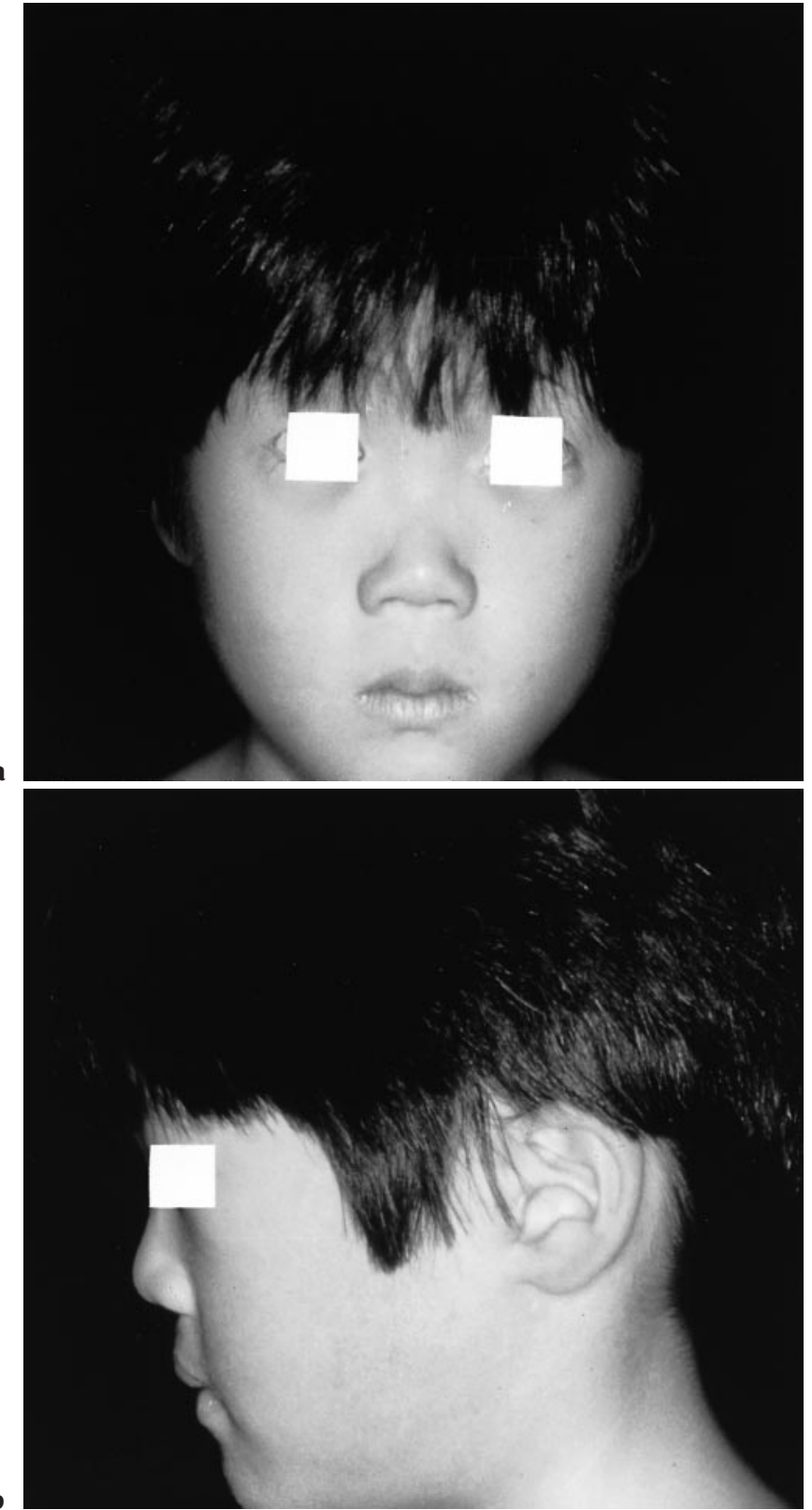

Fig. 1a,b Photographs of the patient (publication permitted by guardian). Note facial dysmorphism, including hypertelorism, mid-face hypoplasia, malformed auricles, and microretrognathia

eral parts of the vitreous bodies, and retinal degeneration in the posterior pole, were evident (Fig. 3). Intraocular pressure was not increased. Otologic examination results were unremarkable. Urinalysis demonstrated microscopic hematuria and mild proteinuria. Renal function was not impaired. Excretory urography and renal ultrasonography yielded normal findings, and further renal evaluation was not carried out. Other laboratory examinations were not contributory. Chromosome analysis showed a normal

Fig. 2a,b Photographs of patient. Note genu recarvatum and hyperextensibility of the wrist
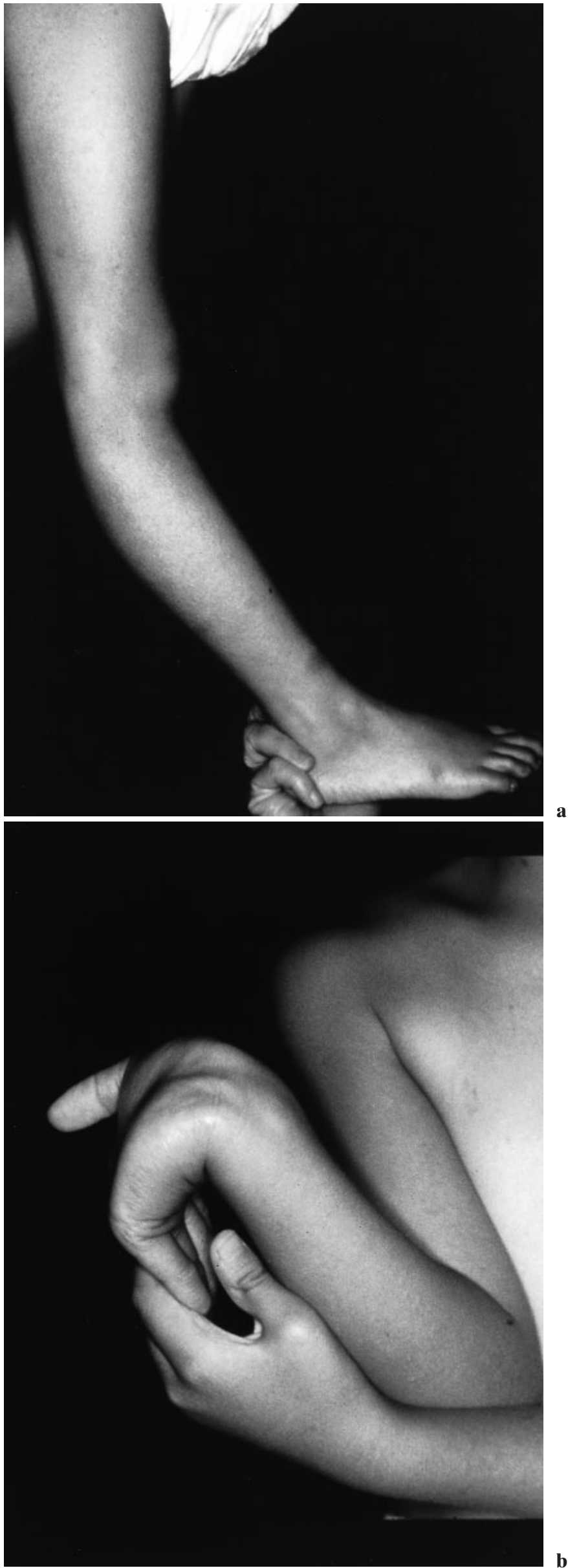


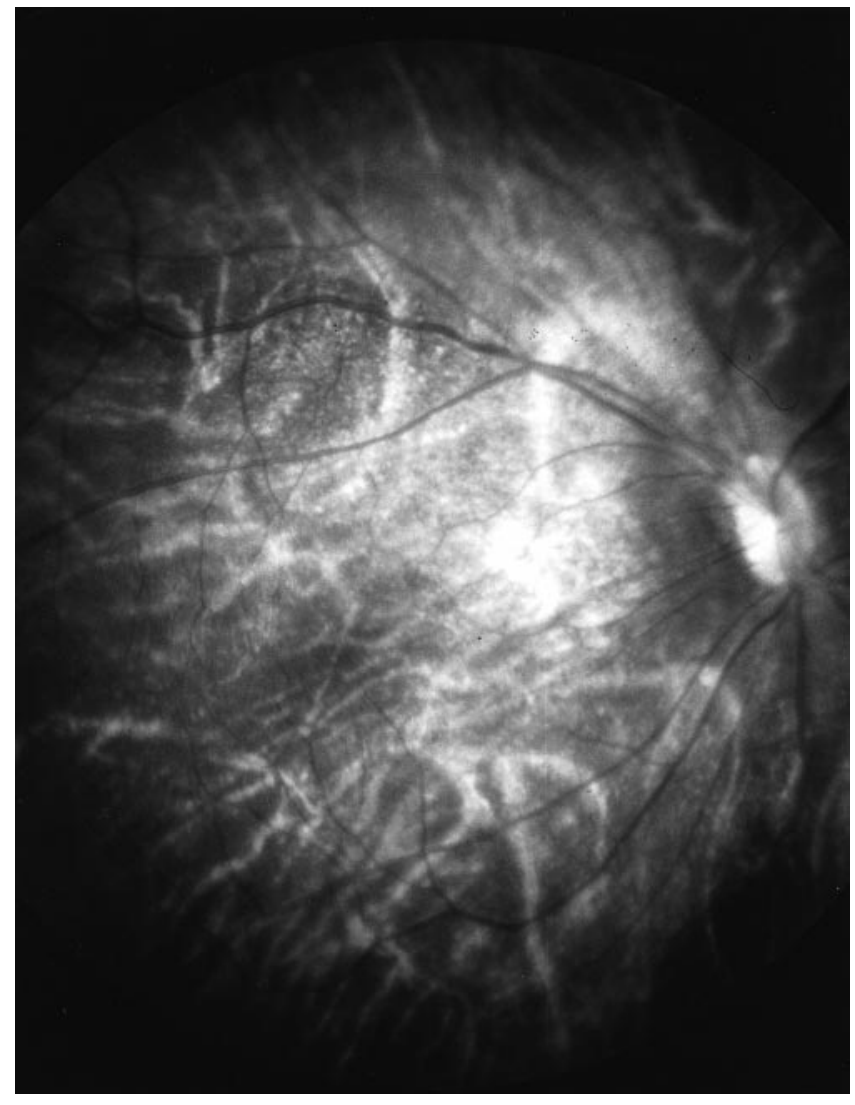

Fig. 3 Photograph of funduscopy. Note retinal degeneration

karyotype. Skeletal survey disclosed multiple wormian bones around the lamboid sutures, hypoplastic facial bones and mandible, narrow thorax with wavy ribs, narrow ilia, and coxa valga with mild broadening of the proximal femora (Fig. 4a,b,c). Histological examination of the skin biopsy sample, including scanning electron microscopy, demonstrated a normal arrangement of the elastic and collagenous fibers in the dermis. Analysis by sodium dodecylsulfate (SDS)-polyacrylamide gel electrophoresis of collagen extracted from skin did not demonstrate evidence of type I collagen abnormality (data not shown). At age 7 years, height was $125.7 \mathrm{~cm}(+1.4 \mathrm{SD})$ and weight $23.8 \mathrm{~kg}$ (+0.4 SD). Microscopic hematuria continued, but renal function was normal. Blood pressure was $92 / 56 \mathrm{mmHg}$.

\section{Discussion}

The present patient exhibited a unique constellation of malformations, comprising joint hyperextensibility, vitreoretinal degeneration with cataracts, facial dysmorphism, and generalized skeletal alterations. The skeletal alterations were individually mild, but constituted an essential part of the syndrome in combination. It remained speculative whether the renal disorder in our patient represented a syndromic constituent or a coincidental abnormality. A literature review and computer-aided diagnostic system of congenital malformation syndromes (Pictures of Standard Syndromes and Undiagnosed Malformations, London Dysmorphology Database, and On-line McKusick Inheritance in Man) failed to disclose any known entities that possess this combination of abnormalities. However, several disorders are worthy of discussion in the differential diagnosis of the present disorder.

The spectacular joint laxity in the present patient initially prompted us to suspect Ehlers-Danlos syndrome (EDS); nevertheless, the absence of skin hyperextensibility, lack of fragility of the skin and other soft tissues, and normal histologic findings of the skin, along with distinct facial dysmorphism and generalized skeletal alterations, eventually precluded most subtypes of EDS (Pope and Smith, 1995). Retinal abnormalities, including retinal detachment and retinal pigment spots as residua of microhemorrhage, rarely occur in EDS, and they are ascribed to tissue fragility, but not to vitreoretinal degeneration. Some patients with EDS VIIA or VIIB exhibit facial dysmorphism resembling that in our patient. In our patient, however, gelelectrophoresis of skin collagen did not reveal any evidence of type I collagen abnormality that is requisite for the diagnosis of EDS VIIA and VIIB.

Hereditary vitreoretinal degeneration is a cardinal feature of type II collagenopathies, including Wagner syndrome, Stickler dysplasia, Kniest dysplasia, and spondyloepiphyseal dysplasia congenita (Korkko et al. 1993). Among these entities, the clinical and radiologic phenotype in Stickler dysplasia considerably overlaps with that of our patient, and significant phenotypic variability in Stickler dysplasia may make the differential diagnosis difficult. However, unlike Stickler dysplasia with mild spondyloepiphyseal dysplasia, neither spondylar modification nor epiphyseal dysplasia was found in our patient, although broadening of the proximal femora in our patient mimicked that of Stickler dysplasia. The joint laxity in our patient was too salient to consider Stickler dysplasia. In general, mid-face hypoplasia in Stickler dysplasia is so conspicuous that it is not comparable to the mild mid-face hypoplasia in our patient. Moreover, unlike findings in Stickler dysplasia, hearing disorder was not found in our patient.

Ter Haar syndrome is a rare multi-system disorder inherited as an autosomal recessive trait (ter Haar et al. 1982; Hamel et al. 1995). The facial dysmorphism and skeletal alterations in ter Haar syndrome resemble those in our patient, and joint laxity is a feature in this entity. However, the ophthalmologic abnormality in ter Haar syndrome, megalocornea with glaucoma and cloudy cornea, is discriminative from the vitreoretinal degeneration in our patient. Furthermore, other major features in ter Haar syndrome, including scoliosis, camptodactyly, and clubfeet, were not found in our patient.

Cantu syndrome shares cataracts, pigmentary abnormality of the macula, joint laxity, and mid-face hypoplasia with micrognathia with the features seen in our patient (Cantu et al. 1997). However, the spectacular mid-face hypoplasia in Cantu syndrome is not comparable to the mild mid-facial recession in our patient. Micromelic short stature and 


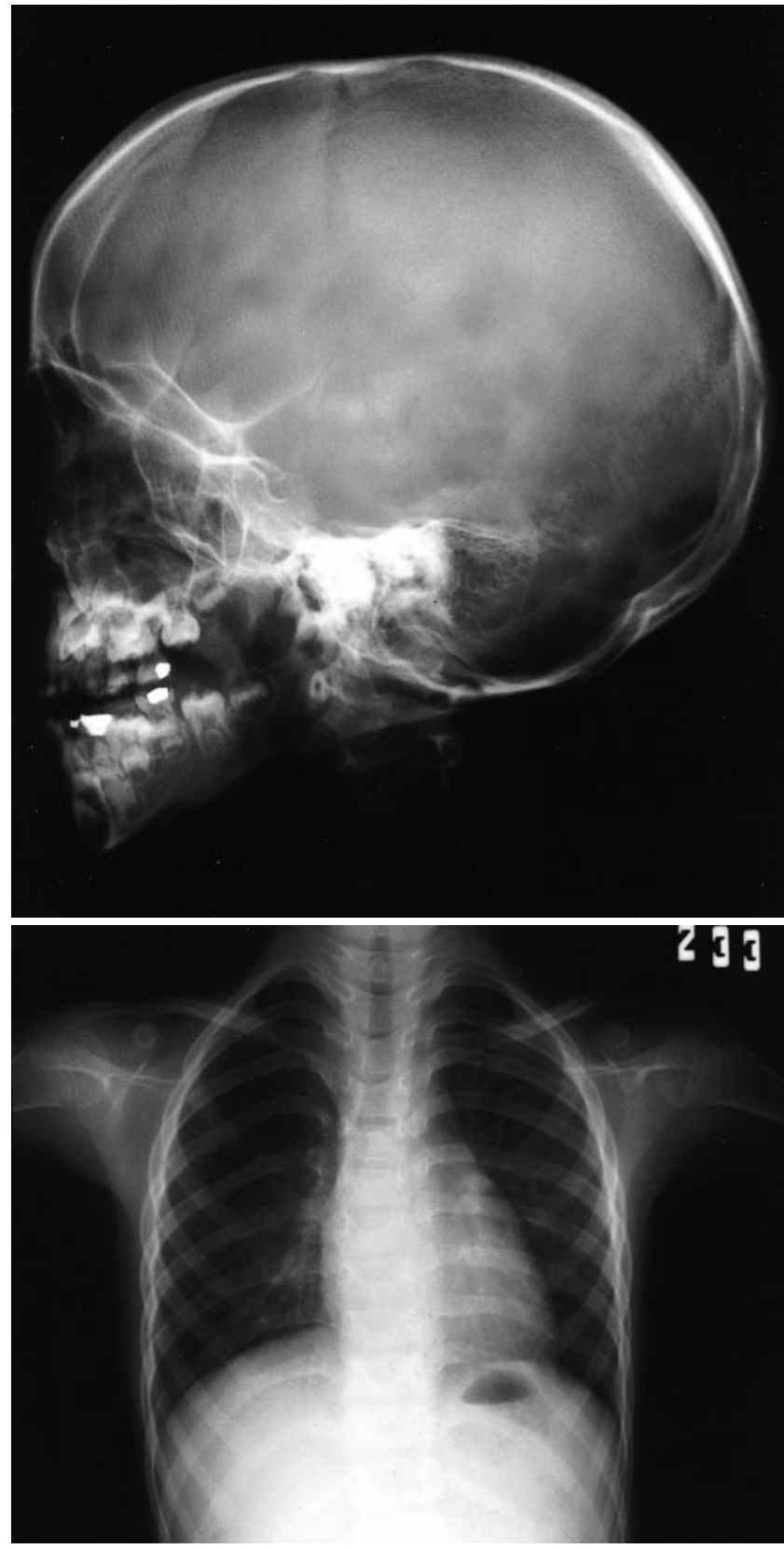

b

severe mental retardation in Cantu syndrome were not features of the present disorder.

In summary, the syndromic constellation of the present disorder does not fit any known congenital malformation syndromes or connective tissue disorders. The etiology remains unknown; nevertheless, it is tempting to speculate that an underlying connective tissue defect may have caused the combination of malformations in the present patient.

\section{References}

Cantu JM, Hernandez A, Armendares S (1997) Case report 58. Synd Ident 5: 22-24

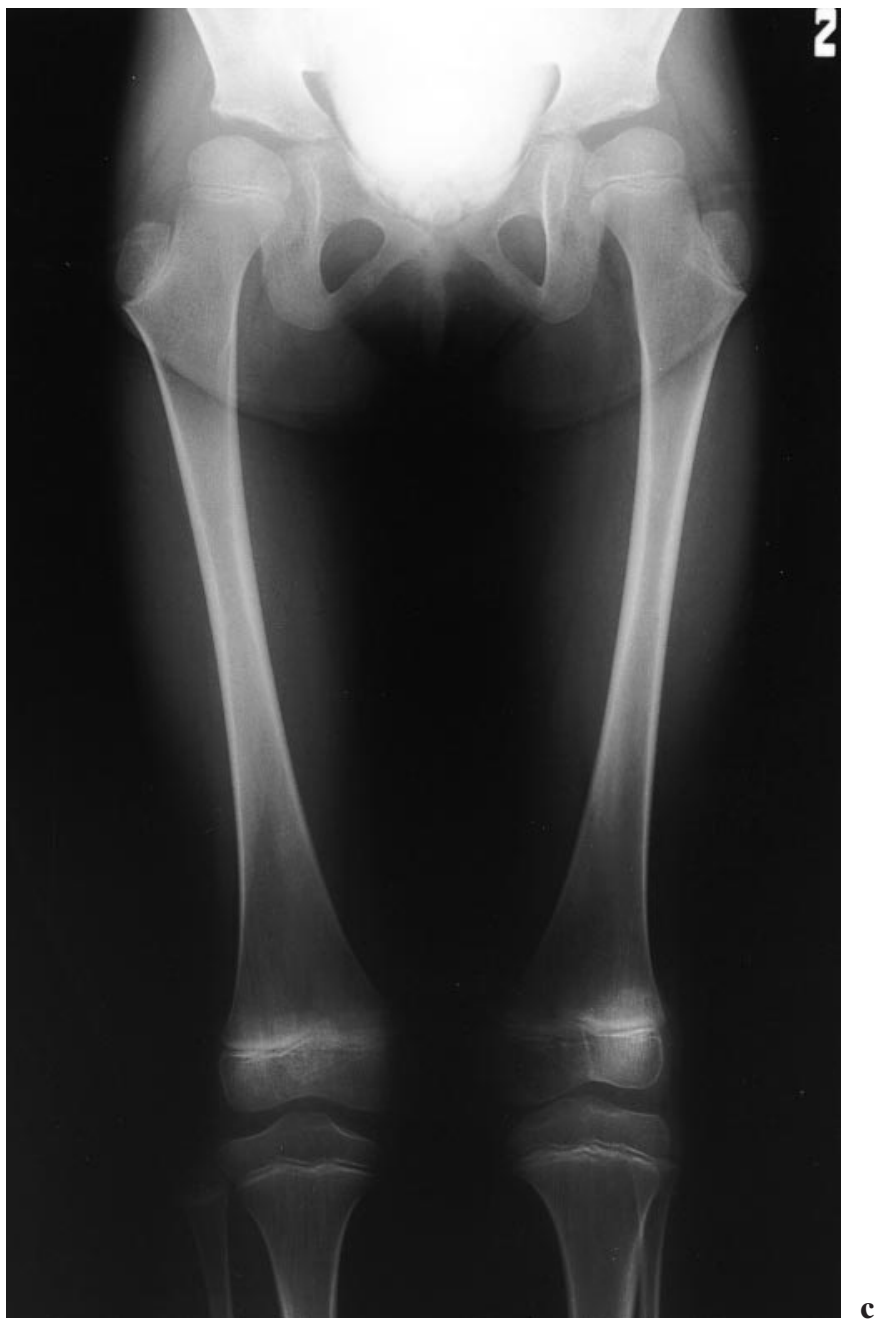

Fig. 4a-c Radiographs of the skull, chest, and lower limbs. Note wormian bones, hypoplastic facial bones and mandible, narrow thorax with wavy ribs and broad proximal femora with coxa valga

Hamel CJ, Draaisma JMT, Pinckers AJLG, Boetes C, Hoppe RLE, Ropers H-H, Brunner HG (1995) Autosomal recessive Melnick-Needles syndrome or ter Haar syndrome? Report of a patient and reappraisal of an earlier report. Am J Med Genet 56: 312-316

Korkko J, Ritvaniemi P, Haataja L, Kaariainen H, Kivirikko KI, Prockop DJ, Ala-Kokko L (1993) Mutation in type II procollagen (COL2A1) that substitutes aspartate for glycine alpha-I-67 and that causes cataracts and retinal detachment: Evidence for molecular heterogeneity in the Wagner syndrome and the Stickler syndrome (arthro-ophthalmopathy). Am J Hum Genet 53: 55-61

Pope FM, Smith R (1995) Color atlas of inherited connective tissue disorders. Mosby-Wolfe, London

Ter Haar B, Hamel B, Hendriks J, de Jager J (1982) Melnick-Needles syndrome: Indication for an autosomal recessive form. Am J Med Genet 13: 469-477 